\title{
Fuzzy based Approach for Determination of Speed in Ad-Hoc Network using Packet Drop
}

\author{
A. S. Mulla \\ Dept. of Computer Applications, \\ Bharati Vidyapeeth Deemed University \\ I.M.R.D.A., Sangli. (MS)
}

\author{
B. T. Jadhav, $\mathrm{PhD}$ \\ Research Guide and Associate Professor \\ Dept. Of Electronics and Computer Science, \\ Yashwantrao Chavan Institute of Science, \\ Satara. (MS)
}

\begin{abstract}
In ad-hoc network, mobile nodes are free to move. They can join or leave the network at any moment of time. Due to this dynamicity, working of network may gets disturbed affecting network performance in terms of throughput, delay, fairness. Considering this we have to focus on various parameters of mobile node. This paper focused on speed and packet drop of mobile nodes. Obtained result is useful for measuring the performance of network.
\end{abstract}

\section{Keywords}

ad-hoc, delay, mobile, mediators, packet drop.

\section{INTRODUCTION}

Mobile ad-hoc network works on the basis of demand of mobile nodes which works with protocols. Mobility, dynamicity nature of nodes may disrupt normal routine of network. As any node can join or leave the network at any instance of time as they are free to move or can be static inside the network. It badly affects normal routine of network causing vulnerability performing malicious activity like packet dropping, energy saving, delay etc. Such problem creates threats for security of network. Regarding this we have analyzed how speed matters on packet dropping. For that we have analyzed data obtained from simulation using ns- 2 .

\section{IMPACT OF PACKET DROPPING ON NETWORK PERFORMANCE}

Packet dropping is a passive attack which can result in repeated retransmissions, which in turn may cause network congestions. A node causing more packet drop will be considered as a malicious node. Along with it if we consider speed as one of parameter. Then we observe that as speed increases, packet drop increases; whereas if speed decreases, packet drop decreases. Keeping slow speed is not feasible for a network. Therefore our proposed work will find the moderate speed where there will be comparatively less packet drop keeping network safe.

To get more accurate result we have applied fuzzy approach.

\section{FUZZY LOGIC}

Fuzzy means not clear or vague data. The Fuzzy Logic tool was introduced in 1965 by Lotfi Zadeh. It is a mathematical tool for dealing with uncertainty.

\section{FUZZY BASED EXPERIMENTAL APPROACH TO DETERMINE SPEED BASED ON PACKET DROP}

Fuzzy based approach considers packet drop as input parameter. The output of linguistic parameter is the speed for mobile node for a given application.
In our case, the rule-base is in a form called functional fuzzy system where each rule $\mathrm{i}$ is written

as follows.

Rule i: IF PktDrop is low THEN Speed = low

To verify its working we will set rule base and verify our result using FIS. Fuzzy controlled packet dropping scheme consists of fuzzification, inference, and defuzzification steps.

\subsection{Structure Of Fuzzy Inference System}

Generally, a fuzzy inference system consists of four modules as shown in Fig.3.

i. Fuzzification converts input which are crisp numbers into fuzzy sets using membership functions.

ii. Knowledge base contains IF-THEN rule.

iii. Inference engine simulates the human reasoning process by making fuzzy inference on the inputs and IF-THEN rules.

iv. Defuzzification converts fuzzy set generated by the inference engine into a crisp value.

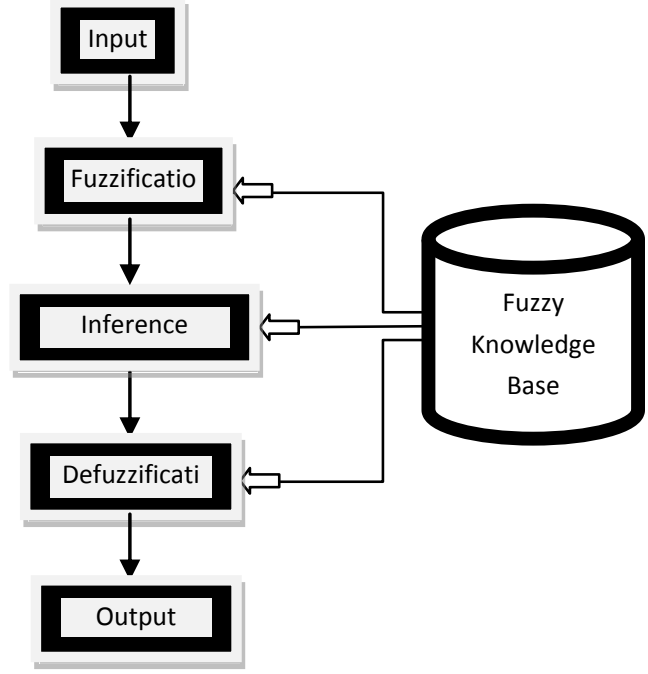

Fig.1: Fuzzy Inference Scheme Diagram

\subsection{Working of FIS}

Following Fig.2 shows our proposed fuzzy based Approach to determine speed based on packet drop.

Here PacketDrop is input parameter and Speed is output parameter. 
For varying number of mediators we have generated different FIS showing different result showing related packet drop and its speed.

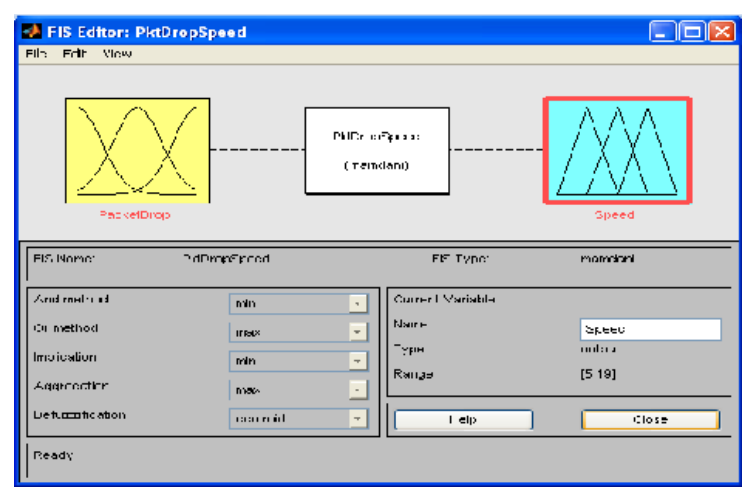

Fig.2: Proposed Working FIS

To verify our result we will categorize input as well as output parameters into different fuzzy set values.

Following Table $1 \& 2$ shows defined fuzzy values for input as well as output parameter. In FIS triangular membership function is used for all criteria for converting the crisp set into fuzzy set.

Table 1. Input Parameter Values

\begin{tabular}{|l|l|l|l|l|l|l|l|l|l|l|l|}
\hline PacketDrop & V & V & L & S & J & M & S & J & H & V & V \\
& V & L & & M & M & & H & H & & H & V \\
& L & & & & & & & & & & H \\
\hline
\end{tabular}

Table 2. Output Parameter Values

\begin{tabular}{|l|l|l|l|l|l|l|l|l|l|l|l|}
\hline Speed & V & V & L & S & J & M & S & J & H & V & V \\
& V & L & & M & M & & H & H & & H & V \\
& L & & & & & & & & & & H \\
\hline
\end{tabular}

\subsection{Working Rule Base}

Practically we observe that for VeryLow PacketDrop, Speed is VeryLow. Whereas for VeryHigh PacketDrop, Speed is VeryHigh. Based on this criteria, we can write distinct fuzzy rules for our FIS as shown below :

i. If PacketDrop is VeryLow then Speed is VeryLow.

ii. If PacketDrop is Low then Speed is Low.

iii. If PacketDrop is Medium then Speed is Medium.

iv. If PacketDrop is High then Speed is High.

v. If PacketDrop is VeryHigh then Speed is VeryHigh.

Rule viewer and surface viewer generated using FIS is as shown in following Figures. Rule Viewer demonstrates if PacketDrop is VeryHigh then Speed is VeryHigh.

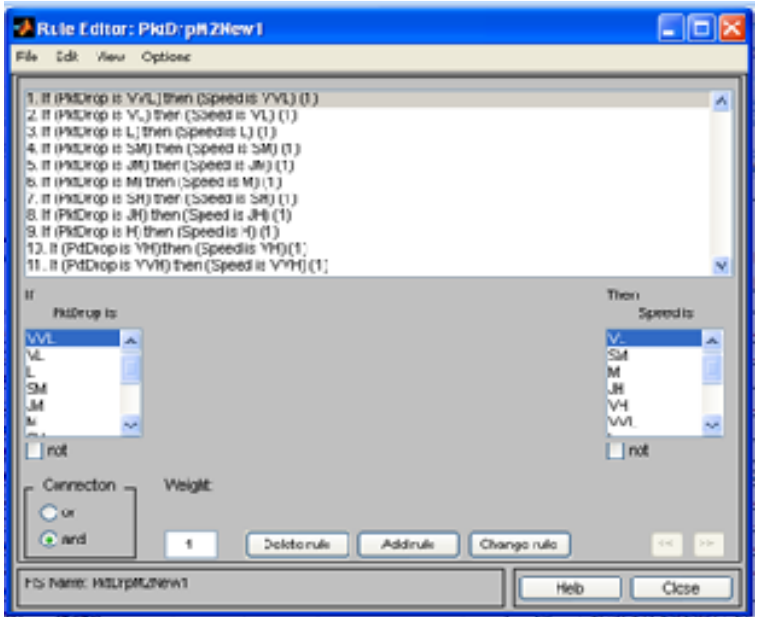

Fig.3: Rule Base

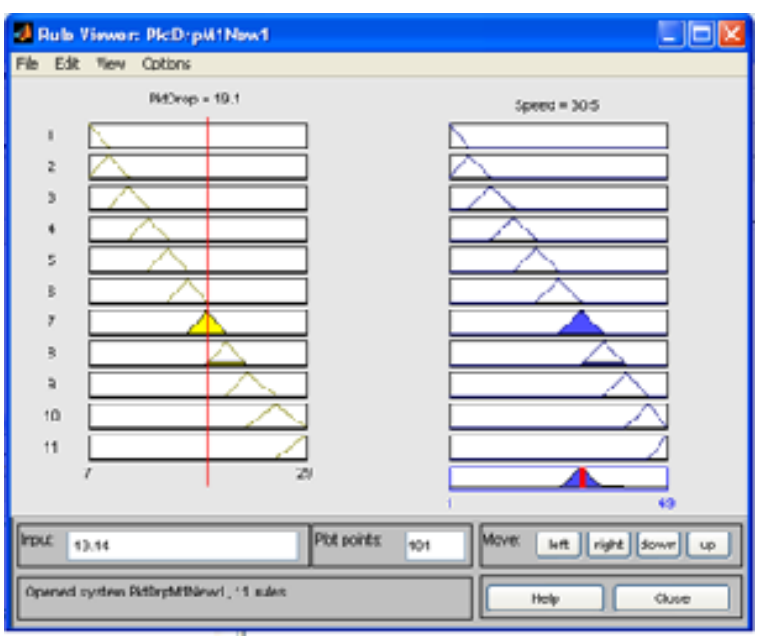

Fig.4: Rule Viewer for One Mediator

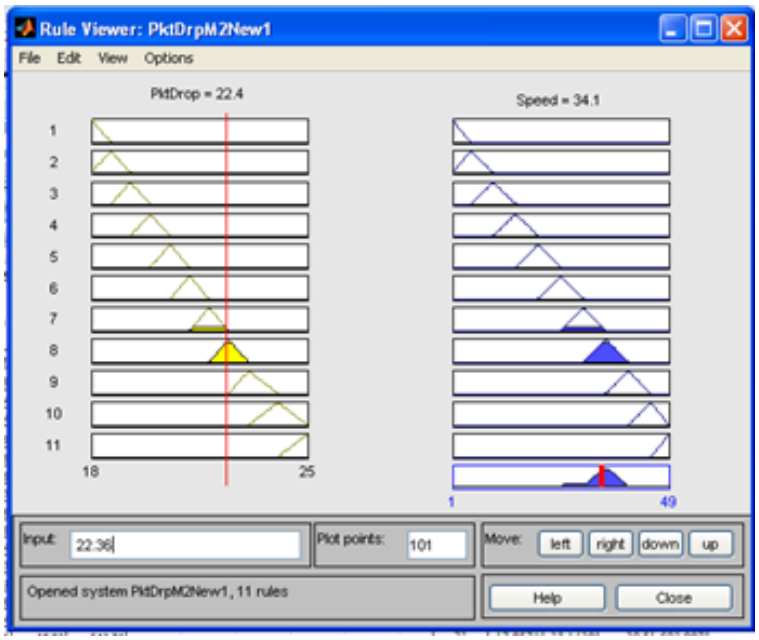

Fig.5: Rule Viewer for Two Mediators 


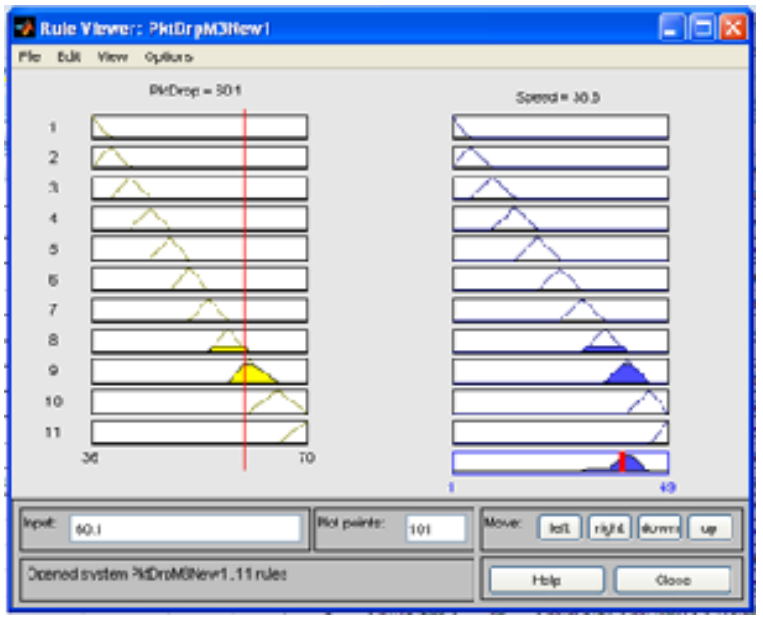

Fig.6: Rule Viewer for Three Mediators

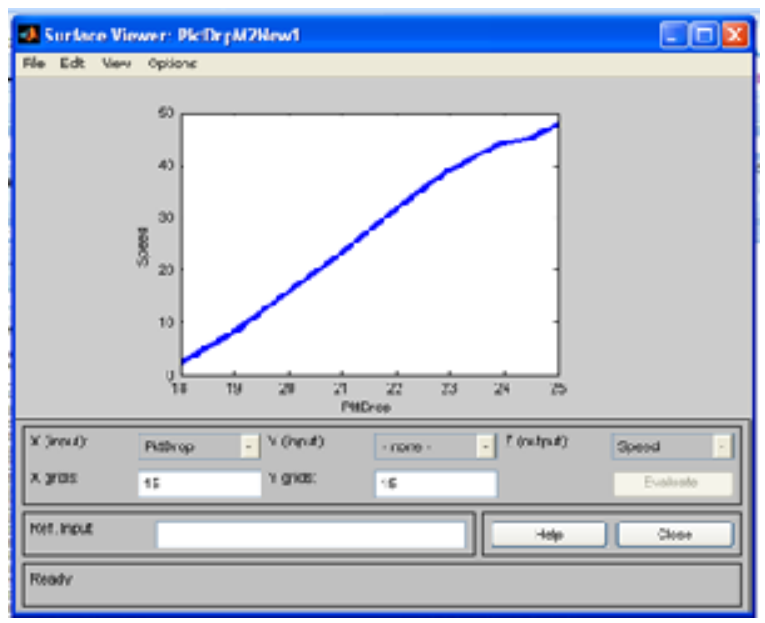

Fig.7: Surface Viewer

Fig. 3 to 7 shows rule base, rule viewer and surface viewer of the same. Thus obtained results (crisp values) from ns-2 is compared with fuzzy values obtained using MATLAB.

Table 3. Comparison between Crisp and Fuzzy Values of Packet Drop and Speed

\begin{tabular}{|c|l|l|l|l|l|}
\hline No. of Mediators & \multicolumn{1}{|c|}{$\begin{array}{c}\text { Packet } \\
\text { Drop }\end{array}$} & Speed & \multicolumn{1}{|c|}{$\begin{array}{c}\text { Packet } \\
\text { Drop }\end{array}$} & $\begin{array}{c}\text { Spee } \\
\text { d }\end{array}$ \\
\cline { 2 - 5 } & \multicolumn{2}{|c|}{ Crisp } & \multicolumn{2}{c|}{ Fuzzy } \\
\hline $\mathbf{1}$ & 19.14 & 29 & 18.914 & 29.7 \\
\hline $\mathbf{2}$ & 22.357 & 37 & 22.171 & 32.7 \\
\hline $\mathbf{3}$ & 60.0952 & 37 & 58.0476 & 35.9 \\
\hline $\mathbf{4}$ & 56.928 & 41 & 56.272 & 39.1 \\
\hline $\mathbf{5}$ & 174.0857 & 39 & 173.5647 & 37.5 \\
\hline
\end{tabular}

Above Table 3 shows comparative values obtained using ns-2 and fuzzy. Result shows us that fuzzy values are more accurate than crisp values. Obtained result is helpful to get absolute values of speed based on packet drop which improves fairness of the network.
Table 4. Observed Throughput Using Fuzzy

\begin{tabular}{|c|l|l|l|l|}
\hline $\begin{array}{c}\text { No. of } \\
\text { Mediato }\end{array}$ & $\begin{array}{c}\text { Packet } \\
\text { Drop }\end{array}$ & Speed & \multicolumn{2}{|c|}{ Throughput } \\
\cline { 2 - 5 } & & & Crisp & Fuzzy \\
\hline $\mathbf{1}$ & 19.14 & 29 & 606.51 & 557 \\
\hline $\mathbf{2}$ & 22.357 & 37 & 636.61 & 624 \\
\hline $\mathbf{3}$ & 60.0952 & 37 & 625.60 & 635 \\
\hline $\mathbf{4}$ & 56.928 & 41 & 546.24 & 489 \\
\hline $\mathbf{5}$ & 174.0857 & 39 & 603.22 & 551 \\
\hline
\end{tabular}

To observe fairness of network in terms of throughput, we have to check database obtained using ns-2

\section{RESULTS AND DISCUSSION}

Above designed scenario shows that as Speed increases, Packet Drop increases and as Speed decreases, Packet Drop decreases.

Increased packet drop affects on network performance in terms of its throughput. Due to which network performance degrades which is not a good practice.

Throughput is one of the parameter of network performance. According to that we have to maintain the speed with packet drop to maximize the throughput with varying number of mediators which will give good result for existing network.

For the same we can compare result obtained using fuzzy in MATLAB. We got above Table 4 which gives us more absolute values of speed, packet drop and throughput. Only maximizing throughput is not our goal. We should maintain the speed with the above specified parameters to carry out safe working of network. Here the obtained speed will be the moderate speed to carry out safe transmission of network.

The same can be applied for varying number of mediators which will give good result for existing network.

\section{CONCLUSION}

Designed fuzzy based system helps the user for decision making while designing one of the secure and safe network according to the above specified scenario with varying number of mediators. Above system will be helpful to get more absolute results for designed scenario which can be further extended to focus on other parameters of safe and secure network.

\section{REFERENCES}

[1] Chonggang Wang,Bo Li, Kazem Sohraby and Yong Peng, An Adaptive Fuzzy-based Control Algorithm for Active Queue Management. IEEE Proc.International Conference on Local ComputerNetworks, pp. 643-649, 2003.

[2] Dong Y.,Makrakis D and Sullivan T, Network Congestioncontrol in ad-hoc IEEE 802.11 Wireless LA N. IEEECanadian conference on electrical and computer Engineering, Vol. 3, pp.1667-1670, 2003.

[3] Janaki Bandara, Resource Allocators for Non Real TimeTraffic in Wireless Sensor Network using Fuzzy Logic. An International Journal on WirelessPersonal Communications, Vol. 21, Issue 3, pp.329-344, June 2002. 
[4] Subasree S and Ravichandran K S, Fuzzy DS RED An Intelligent Active Queue Management Scheme for TCP/IP Diff-Serv. Transaction on Engg.Computing and Technology, pp. 245-248, December, 2004.

[5] T.J. Ross, Fuzzy Logic for Engineering Applications, John Wiley, 1998

[6] Vinod Kumar, "A Fuzzy Based Control over Malicious Nodes in Manet", International Journal of Latest Trends in Engineering and Technology (IJLTET)
[7] Yuan Chen and Lemin Li, A Fuzzy Fair Packet Dropping Algorithm Supporting Differentiated Services. IEEE Proc. 5th International Conference on Computer and Information Technology (CIT'05), 2005.

[8] Yuan Chen and Lemin Li, A Wireless packet dropping algorithm considering fairness and channel condition. An International conference on Communications, circuits and system, Vol.1, pp. 369-373, 2004. 